discrepancies between their work and ours were discussed in a paper from this Laboratory." This paper directs attention to the fact that Jeffery and Vogel used a bridge and cells which, due to parasitic currents, could readily give errors in the results. Their use of a water thermostat leads to additional errors of similar nature, as has been shown by Jones and Josephs. ${ }^{5}$ Jeffery and Vogel have published conductance measurements only. To obtain ion con. ductances transference data are necessary. In their paper they present two sets of figures for the limiting ion conductances, leaving the choice to the reader. One set is based on a limiting transference number of potassium in potassium chloride of 0.497 and the other on a value of that constant of $0 \cdot 490$, the latter being the result of recent work in our Laboratory.

As to our results, the limiting conductance of the chloride ion, $\lambda_{0 \mathrm{Cl}}$, from which all the other limiting ion conductances may be computed, is based on measurements of conductance, and transference numbers, at a series of concentrations of four different chlorides ( $\mathrm{KCl}, \mathrm{NaCl}, \mathrm{LiCl}$, and $\mathrm{HCl}$ ), and the same result $\lambda_{0 \mathrm{Cl}}=76 \cdot 32$, within a few hundredths of a per cent is obtained from the data on each electrolyte. Previous workers have assumed validity of Kohlrausch's law of independent ion mobilities. We feel that our work has proved it, at least for these chlorides.

Our extrapolations have been made using a method based on the assumption that as the dilution is increased ion conductances will approach the relations derived by Onsager. ${ }^{6}$

Vogel and Jeffery also report an ionisation constant of $1.776 \times 10^{-5}$ for acetic acid which, they point out, differs considerably from that published by MacInnes and Shedlovsky ${ }^{2}$ who give $1.753 \times 10^{-5}$ for that constant. We feel that it is sufficient to direct attention to the recent result of Harned and Ehlers ${ }^{8}$ who, as the result of an extensive and careful investigation involving galvanic cells without liquid junctions, obtained the value $1.754 \times 10^{-5}$ This check is particularly gratifying, since the methods used in the two researches are quite different.

DUNCAN A. MacINnes.

ThEODORE SHEDLOVSKY.

LEWIS G. LONGSWORTH.

The Rockefeller Institute

for Medical Research,

New York, N.Y., Sept. 28.

I NATURe, 130, $435 ; 1932$.
$J$. Amer. Chem. Soc., 54, $2758 ; 1932$.

J. Chem. Soc., 1715; 1931. 400; 1932

J. Amer. Chem. Soc., 54, 1411; 1932.

P. Amer. Chem. Soc., 50, 1065; 1928.

Physik. Z., 27, 388 ; 1926. 28, 277 ;
J. Amer. Chem. Soc., 54, 1429; 1932.

7. J. Amer. Chem. Soc., 54, 1429; 1932.

\section{Emission of Positive Ions from Cold Surfaces under the Influence of Strong Electric Fields}

BEAMs ${ }^{1}$ has brought forward experimental evidence showing that the application of a field of the order of $5 \times 10^{6}$ volts per cm. to a tungsten surface may result in the direct removal of adsorbed positive ions of the alkali metals. Ianitsky ${ }^{2}$ had previously described experiments in which such an effect was obtained with fields about a hundred times smaller, the positive ions concerned being those of the permanent gases.

No. 3290 , Vol. 130]
I have recently been led to investigate the fluorescence of the glass in a hot cathode $\mathrm{X}$-ray tube, ${ }^{3}$ and found that the most negative part of the glass occasionally showed fluorescent spots, which must therefore have been due to positive ion bombardment. The phenomenon was observed with the filament switched off, the gas pressure less than $2 \times 10^{-6} \mathrm{~mm}$. of $\mathrm{Hg}$, and only $20 \mathrm{k.v}$. across the tube. A detailed consideration of possible mechanisms of formation of the positive ions eliminated all but one, namely, their removal from an adsorbed layer of impurity on the anode. This confirms the observations of Tanitsky, who however did not observe the positive ions as such but by the resulting change in gas pressure.

Ianitsky suggested that the adsorbed gas layer normally exists in a partially ionised form, so that the phenomenon observed by him and by myself would be fundamentally the same as that observed by Beams. It seems to me more probable, however, that it was fundamentally different, the gas layer being first ionised by $\mathbf{X}$-rays or by electrons (which would have inevitably been present in the experiments), and then the ions removed by the field. Since some of the ions would be formed at a distance of several atomic radii from the surface, a much smaller field would suffice for their removal than was required in Beams's experiments with degassed surfaces. This agrees with experiment.

It seems, however, likely that the compound effect was present in the experiments Beams carried out on gas layers. If this was so, the observed critical voltage for the effect to occur was either that for the positive ion emission or that for starting the discharge responsible for the ionisation, whichever was the larger of the two.

Wills Physics Laboratory,

W. R. HARPER.

University of Bristol.

1 Phys. Rev., 41, 687; 1932

- Described in the October issue of the Proc. Camb. Phil. Soc. ; 1932.

\section{Origin of Zero-Point Entropy}

Eucker and his collaborators have pointed out that the failure of Nernst's heat theorem-the so-called third law of thermodynamics-which occurs in some cases, is due to the zero-point entropy ${ }^{1}$. A definite zero-point entropy arises from the existence of 'frozen-in' phases stable at higher temperatures which do not reach the real thermal equilibrium on cooling to the neighbourhood of the absolute zero. Teske and I, for example, have found that solid carbon monoxide near $10^{\circ} \mathrm{K}$. exhibits such a state in spite of its crystalline structure ${ }^{2}$. Clayton and Giauque have confirmed this result ${ }^{3}$ and explained the discrepancy by the suggestion that the asymmetry of the carbon monoxide molecule gives rise to two positions in the lattice of nearly equal energy. The formation of a fully ordered crystal is thus prevented. No such effect is to be expected in the case of nitrogen, which is a symmetrical molecule. Dr. Teller has directed my attention to the fact that similar behaviour may be shown by nitrous oxide as opposed to the symmetrical carbon dioxide: this has proved to be true.

The following table gives some zero-point entropies calculated from the difference of the direct thermal measurements and the entropy value from band spectroscopic data. If the disarrangement is 